\title{
Outcomes of Ultrasound-Guided Extracorporeal Shock Wave Therapy for Painful Stump Neuroma
}

\author{
Yun Jae Jung, $\mathrm{MD}^{1}$, Won Yong Park, $\mathrm{MD}^{1}$, Jong Hyun Jeon, $\mathrm{MD}^{1}$, Jeong Hyeon Mun, $\mathrm{MD}^{1}$, \\ Yoon Soo Cho, $\mathrm{MD}^{2}$, Ah Young Jun, $\mathrm{MD}^{1}$, Ki Un Jang, MD, $\mathrm{PhD}^{1}$, Cheong Hoon Seo, $\mathrm{MD}^{2}$
}

\begin{abstract}
${ }^{1}$ Department of Rehabilitation Medicine, Dongtan Sacred Heart Hospital, Hallym University College of Medicine, Hwaseong; ${ }^{2}$ Department of Rehabilitation Medicine, Hangang Sacred-Heart Hospital, Hallym University College of Medicine, Seoul, Korea
\end{abstract}

Objective To investigate the effect of extracorporeal shock wave therapy (ESWT) on painful stump neuroma. Methods Thirty patients with stump neuroma at the distal end of an amputation site were assigned randomly to the ESWT group $(n=15)$ and the transcutaneous electrical nerve stimulation (TENS)+desensitization+pharmacological treatment group $(n=15)$. For 3 weeks, the ESWT group received a weekly session involving 1,500 pulses at 0.10 $\mathrm{mJ} / \mathrm{mm}^{2}$, while the control group was treated 10 times each, 40 minutes per day with TENS and desensitization treatment, and daily medication for 3 weeks. ESWT stimulation was given by focusing on the area at the neuroma site clearly identified by ultrasound.

Results The changes in the McGill pain questionnaire were 38.8 \pm 9.0 prior to treatment and $11.8 \pm 3.1$ following the treatment. The corresponding values for the control group were $37.2 \pm 7.7$ and $28.5 \pm 10.3$. The changes between groups were significantly different $(\mathrm{p}=0.035)$. The change in visual analog scale prior to and after treatment was $7.0 \pm 1.5$ and $2.8 \pm 0.8$ in the ESWT group, respectively, and 7.2 \pm 1.4 and $5.8 \pm 2.0$ in the control group. These changes between the groups were also significantly different $(\mathrm{p}=0.010)$. The outcome in the pain rating scale also showed significant differences between groups $(\mathrm{p}<0.001)$. Changes in neuroma size and pain pressure threshold $\left(\mathrm{lb} / \mathrm{cm}^{2}\right)$ were not significantly different between groups ( $p>0.05)$.

Conclusion The study findings imply that ESWT for stump neuroma is superior to conventional therapy.

Keywords Amputation stumps, Neuroma, Extracorporeal shock wave therapy, Ultrasonography, Visual analogue scale

\footnotetext{
Received April 3, 2014; Accepted June 16, 2014

Corresponding author: Cheong Hoon Seo

Department of Rehabilitation Medicine, Hangang Sacred-Heart Hospital, Hallym University College of Medicine, 12 Beodeunaru-ro 7-gil, Yeongdeungpo-gu, Seoul 150-719, Korea

Tel: +82-2-2639-5730, Fax: +82-2-2635-7820, E-mail: s9036@naver.com

(c) This is an open-access article distributed under the terms of the Creative Commons Attribution Non-Commercial License (http://creativecommons. org/licenses/by-nc/3.0) which permits unrestricted noncommercial use, distribution, and reproduction in any medium, provided the original work is properly cited.

Copyright $\odot 2014$ by Korean Academy of Rehabilitation Medicine
}

\section{INTRODUCTION}

Neuroma was first described by Odier in 1811 as an extremely sensitive bulbous stump of a transected nerve [1]. Neuromas either develop as part of a normal reparative process after nerve injury or as a result of chronic irritation, pressure, stretch, poor repair of nerve lesions or previous neuromas, laceration, crush injury, or blunt trauma to nerves [2]. They typically develop approximately 6 to 10 weeks after trauma with most presenting within 1 to 12 months after injury or surgery [2]. 
Neuroma that forms at the cut end of a nerve is a major cause of stump pain [3]. A stump neuroma is an outgrowth from a severed nerve that takes the form of a ball or stump. Substantial pain associated with a scar and altered sensibility in the distribution of the involved nerve is the clinical hallmark of a neuroma [4]. Patients usually describe the pain as a low-intensity dull pain or intense paroxysmal burning pain, often triggered by external stimuli like touch and temperature. Tinel sign is often positive and may be associated with an area of hyperalgesia and allodynia. Numerous types of nerve injuries can lead to the formation of a stump neuroma and treatment options are available for patients with this condition. Attempts to prevent neuroma formation have been uniformly frustrating, which is reflected in the myriad methods described, both pharmaceutical and surgical, including implantation into muscle or bone, nerve to nerve repair, capping with inert materials, cauterization, steroid, phenol, and alcohol injections [5]. Surgical resection of neuroma often results in only a short duration of pain relief, and usually is followed by redevelopment of neuromas and related pain [6].

Extracorporeal shock wave therapy (ESWT) is a more recently developed treatment for diseases of the musculoskeletal system. ESWT is effective in treatment of musculoskeletal diseases, such as nonunion of pseudoarthrosis or fracture, calcific tenosynovitis, and plantar fasciitis. However, the effectiveness of ESWT to treat painful stump neuroma has not been reported. Improved effectiveness in other diseases is thought to stem from the promotion of angiogenesis, increasing perfusion in ischemic tissues, decreasing inflammation, enhancing cell differentiation, accelerating wound healing, and alleviation of pain by altering pain signal [7]. Considering these pathophysiologies, we hypothesized that ESWT could reduce the symptom of painful stump neuroma.

Electromagnetic generation of ESWT coupled with ultrasound (US) visualization is now available, but has not been as thoroughly evaluated. The purpose of this study was to evaluate the effectiveness of electromagnetically generated, US-guided ESWT in the treatment of painful stump neuroma.

\section{MATERIALS AND METHODS}

Patients who underwent amputation surgery at a university clinic from 2010 to 2012 were the subjects of this study. Patients diagnosed only as stump neuroma were included. Exclusion criteria included stress or overt stump site fracture, overlying infection or cellulitis, im-

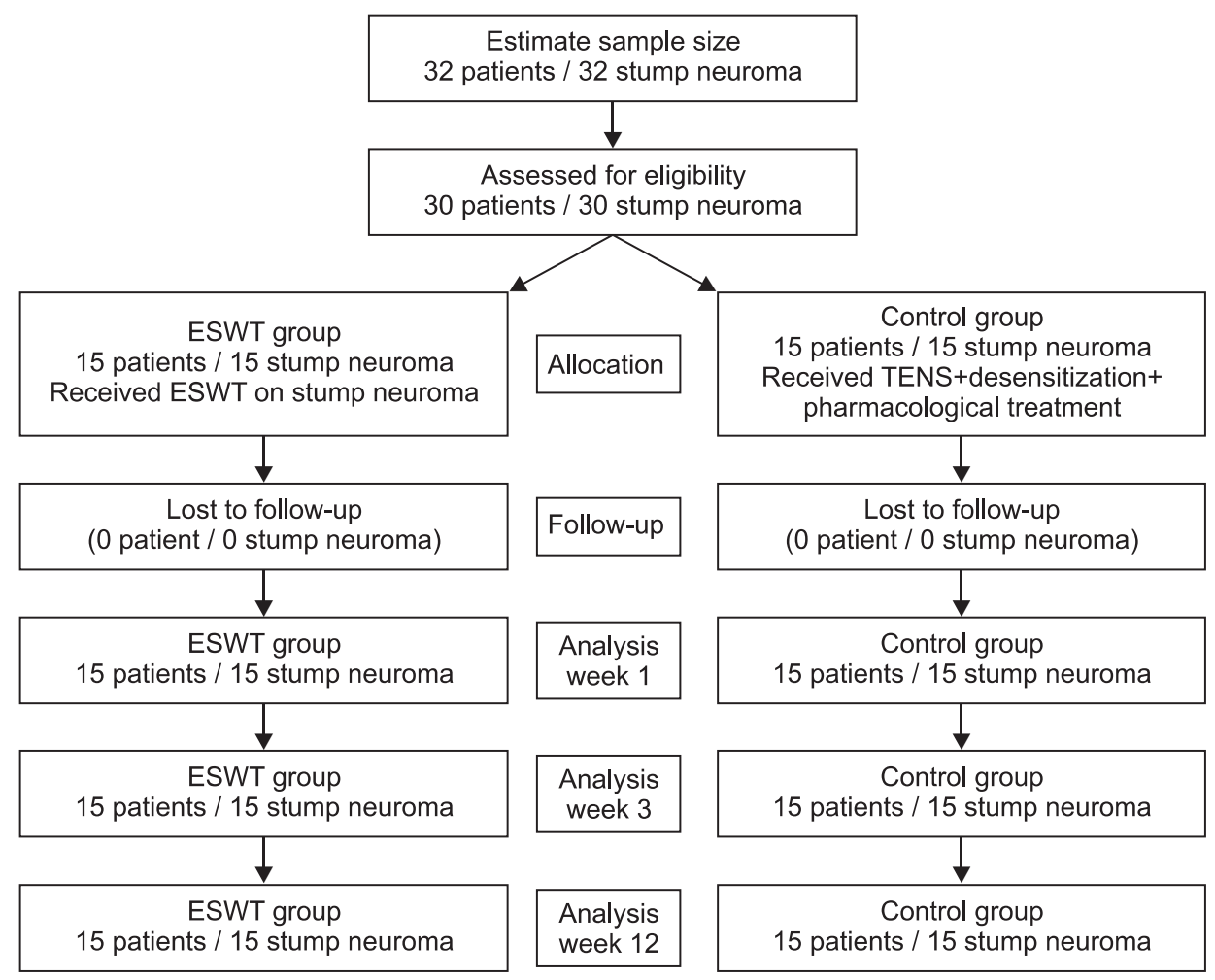

Fig. 1. Flow chart of extracorporeal shock wave therapy (ESWT) for stump neuroma. TENS, transcutaneous electrical nerve stimulation. 
mature stump site condition, and absence of well-defined neuroma by US. Diagnosis of stump neuroma was made based on the patient's history of neuropathic pain, physical findings of pain on palpation of stump site and detected by US as a well-defined hypoechoic mass.

This study was a prospective, randomized experimental and control group research with the subjects divided into two groups: an experimental group of 15 subjects who underwent ESWT and a control group of 15 subjects who underwent transcutaneous electrical nerve stimulation (TENS) along with a desensitizing technique and pharmacological treatment (Fig. 1). Each stump site was subjected to a single ESWT session using an EvoTron RFL0300 device (SwiTech Medical AG, Kreuzlingen, Switzerland). ESWT was performed by a physician who was not involved in the selection of the patients and was not aware of the measurement scale. The scale was administered and assessed before each session by an observer. Prior to ESWT, the point of maximal tenderness was palpated and marked with a skin marker. The ESWT probe was aimed at the point of maximal tenderness and the US screen was used to visualize an oval, hypoechoic mass that may be well-defined, larger than the diameter of the nerve, and in direct contiguity with the nerve. The marker area was pressed with a linear transducer $(10 \mathrm{MHz})$ in real-time to assess whether skin marker area matched the neuroma location identified with US and the compression of neuroma was monitored to assess whether maximum tenderness was triggered. On US, normal nerves appear as cord-like structures composed of echogenic linear striations on longitudinally oriented images and round echogenic structures on transverse images. For ESWT treatment, subjects were given total of 1,500 shock waves for each treatment at the rate of 240 waves, each with low energy flux density (EFD) of $0.10 \mathrm{~mJ} / \mathrm{mm}^{2} / \mathrm{min}$, with a 1-week interval between treatments (total of 4,500 shock waves). TENS and desensitization treatments were given 10 times in a week with a duration of 20 minutes for each treatment and daily pharmacological treatment comprising pelubiprofen $30 \mathrm{mg}$ three time a day and ibuprofen $30 \mathrm{mg}$ three time a day. Both groups underwent treatment for 3 weeks.

Subjects were assessed by an observer prior to the ESWT sessions. The effectiveness of ESWT was measured by the McGill pain questionnaire, visual analogue scale (VAS), pain rating scale (PRS), neuroma size, and pain threshold $\left(\mathrm{lb} / \mathrm{cm}^{2}\right)$, and evaluated by BASELINE (Hoggan Health Industries, West Jordan, UT, USA). Each scale was assessed prior to the first treatment (initial assessment), 1 week after the first treatment, 1 week after the third treatment, and 3 months after the third treatment (final assessment) as the evaluation method for the effectiveness of treatment. The pain from stump neuroma can be position- or movement-related, is exacerbated by a range of physical factors, and seems to be more intense in the movement [8]. Thus, active and resting state measurements were performed to measure VAS and PRS, respectively, and VAS-R (resting status), VAS-A (active status), PRS-R (resting status), and PRS-A (active status) were measured from the initial to final assessments to find out whether treatment effect occurs with both active and resting status. Examination using an ultrasonography 128 BW device (Medison, Seoul, Korea) revealed a discrete hypoechoic mass in the distal stump, directly continuous to the posterior tibial nerve (Fig. 2), consistent with ultrasonographic findings of neuroma. Both transverse plane and longitudinal plane examinations were performed to measure neuroma size, which were done by measuring the largest area from the transverse plane towards the nerve movement direction.

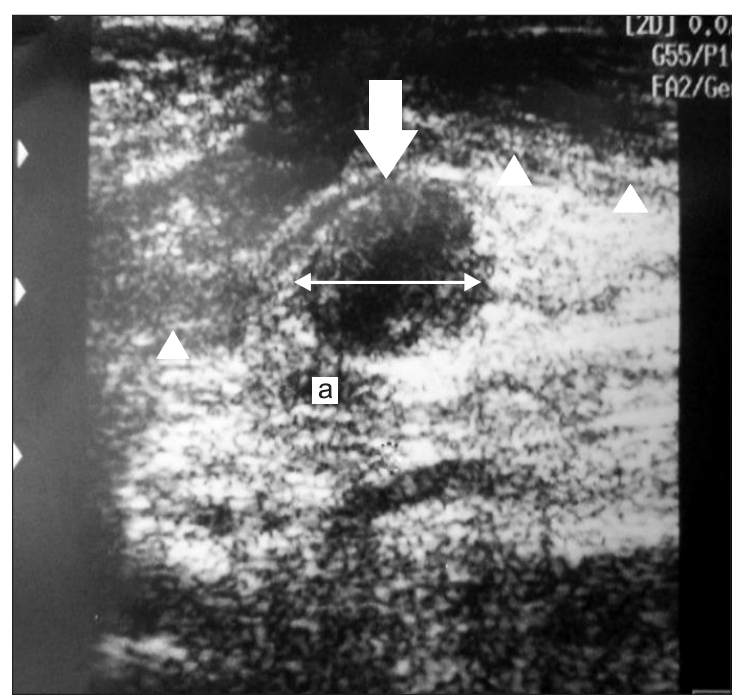

Fig. 2. Transverse ultrasound image of medial ankle reveals ovoid, hypoechoic mass (arrow), directly continuous to the posterior tibial nerve. Posterior tibial neuroma is close to the posterior tibial artery (a) and covered by flexor retinaculum (arrowhead). Digital calipers indicate the size of the neuroma to be $16.1 \mathrm{~mm}$. 
Collected data were analyzed using the SPSS ver. 21.0 (IBM, Armonk, NY, USA). Fisher exact test and MannWhitney test were carried out for homogeneity testing on the two groups. Since the results of review of the normality assumption on the preliminary assessment tools of the two groups was satisfactory, Mann-Whitney test was carried out to verify the preliminary homogeneity and repeated measure ANOVA was carried out to examine the effectiveness of the results of preliminary assessment, and assessment following the first and third treatments.

Prior to execution of repeated measure ANOVA, the Mauchly's sphericity test for measurement of homoscedasticity and compound symmetry was performed. In the case of satisfaction of the sphericity assumption ( $>0.05)$, verification of time effect (time) and reciprocal action effect (group $\times$ time) were analyzed using sphericity assumption statistics of the univariate test. If sphericity assumption was not satisfied ( $\mathrm{p}<0.05)$, statistics of Wilks' lambda in the multivariate test was used.

Table 1. Demographic and clinical characteristics of patients

\begin{tabular}{llll}
\hline \multicolumn{1}{c}{ Characteristic } & ESWT & Control & p-value \\
\hline Sex & & & 0.759 \\
\hline Male & $14(93.3)$ & $14(93.3)$ & \\
\hline Female & $1(6.7)$ & $1(6.7)$ & \\
Age & $45.2 \pm 8.9$ & $41.7 \pm 9.9$ & 0.217 \\
\hline Amputation site & & & 0.509 \\
\hline Lt. 1st thumb & $0(0)$ & $1(6.7)$ & \\
\hline Lt. 2nd, 5th finger & $1(6.7)$ & $0(0)$ & \\
\hline Lt. shoulder dislocation & $1(6.7)$ & $2(13.3)$ & \\
\hline Lt. transhumeral & $0(0)$ & $1(6.7)$ & \\
\hline Lt. transradial & $3(20)$ & $0(0)$ & \\
\hline Lt. transtibial & $2(13.3)$ & $1(6.7)$ & \\
\hline Rt. 1st thumb & $0(0)$ & $1(6.7)$ & \\
\hline Rt. 1st toe & $1(6.7)$ & $1(6.7)$ & \\
\hline Rt. 2nd, 3rd finger & $0(0)$ & $1(6.7)$ & \\
\hline Rt. shoulder dislocation & $2(13.3)$ & $0(0)$ \\
\hline Rt. transhumeral & $1(6.7)$ & $2(13.3)$ & \\
\hline Rt. transradial & $3(20)$ & $5(33.3)$ & \\
\hline Rt. transtibial & $1(6.7)$ & $0(0)$ \\
\hline
\end{tabular}

Values are presented as mean \pm standard deviation or number (\%).

ESWT, extracorporeal shock wave therapy; Lt., left; Rt., right.

\section{RESULTS}

\section{Patient demographic and clinical characteristics}

The total number of subjects was 30 (28 males and 2 females). Of the 15 subjects in the experimental group, 14 were male and 1 was female, while the control group was composed of 14 males and 1 female. The average age was $45.2 \pm 8.9$ years for the experimental group and $41.7 \pm 9.9$ years for the control group. Physical examination involving palpation of a discrete area of tenderness resulted in distally radiating pain in the distribution of the peripheral nerve plus the identification of an area distal to the site of the positive Tinel sign that had altered sensation (hypoesthesia, hyperalgesia, or anesthesia) in the distribution of the involved nerve in both the experimental group and the control group. Fisher exact test and MannWhitney test were carried out to examine the preliminary homogeneity between the two groups; the groups were homogeneous as there was no significant difference ( $p>0.05$ ) (Table 1). The Mann-Whitney test carried out to examine the preliminary homogeneity of the preliminary assessment tools also revealed homogeneity $(\mathrm{p}>0.05)$ (Table 2).

\section{Change of McGill pain questionnaire}

The sphericity assumption was satisfied as a result of repeated measure ANOVA on the McGill pain questionnaire $(p=0.542)$. There was a significant difference in the pain questionnaire results between the groups $(\mathrm{p}=0.035)$, with time $(\mathrm{p}<0.001)$, and with reciprocal action $(\mathrm{p}=0.001)$. Contrast test according to time revealed a reduction following the first and third treatments in comparison

Table 2. Pre-homogeneity test results

\begin{tabular}{lrrc}
\hline & ESWT & Control & p-value \\
\hline Neuroma size & $1.9 \pm 0.4$ & $1.9 \pm 0.4$ & 0.624 \\
McGill pain questionnaire & $31.2 \pm 7.3$ & $32.2 \pm 9.5$ & 0.935 \\
VAS-R & $6.0 \pm 1.9$ & $6.4 \pm 1.7$ & 0.653 \\
VAS-A & $7.0 \pm 1.5$ & $7.2 \pm 1.4$ & 0.713 \\
Pain threshold $\left(\mathrm{Ib} / \mathrm{cm}^{2}\right)$ & $7.3 \pm 2.8$ & $7.0 \pm 2.5$ & 0.838 \\
PRS-R & $38.2 \pm 15.5$ & $39.4 \pm 15.6$ & 0.870 \\
PRS-A & $43.3 \pm 12.4$ & $44.2 \pm 15.4$ & 0.744 \\
\hline
\end{tabular}

Values are presented as mean \pm standard deviation. ESWT, extracorporeal shock wave therapy; VAS, visual analogue scale; R, resting status; A, active status; PRS, pain rating scale. 
to prior to commencement, indicating a significant reduction in pain evident by responses in the McGill pain questionnaire as the treatment was carried out. A significant difference in the McGill pain questionnaire results was evident between the groups. There was also a significant difference in the results of the contrast test on reciprocal action prior to commencement and following the third treatment, indicating that, although the McGill pain questionnaire results prior to commencement were higher in the ESWT group, the results were lower in the ESWT group following the third treatment and that ESWT had greater effectiveness after the third treatment (Table $3)$.

Table 3. Change in McGill pain questionnaire scores

\begin{tabular}{lcccc}
\hline & ESWT & Control & F & p-value \\
\hline Before first therapy & $38.8 \pm 9.0$ & $37.2 \pm 7.7$ & - & - \\
After first therapy & $31.5 \pm 8.5$ & $32.1 \pm 8.7$ & - & - \\
After third therapy & $20.7 \pm 8.1$ & $26.2 \pm 7.9$ & - & - \\
Final assessment & $11.8 \pm 3.1$ & $28.5 \pm 10.3$ & - & - \\
Group (G) & - & - & 15.8 & $0.035^{*}$ \\
Time (T) & - & - & 73.7 & $<0.001^{*}$ \\
G×T & - & - & - & $0.001^{*}$ \\
Mauchly's sphericity test & & W=0.224 & & 0.542 \\
\hline
\end{tabular}

Values are presented as mean \pm standard deviation.

ESWT, extracorporeal shock wave therapy.

${ }^{*} \mathrm{p}<0.05$.

Table 4. Change of VAS of resting and active status

\begin{tabular}{|c|c|c|c|c|}
\hline & ESWT & Control & $\mathbf{F}$ & p-value \\
\hline \multicolumn{5}{|l|}{ VAS-R } \\
\hline Before first therapy & $6.0 \pm 1.9$ & $6.4 \pm 1.7$ & - & - \\
\hline After first therapy & $5.1 \pm 1.9$ & $5.4 \pm 2.1$ & - & - \\
\hline After third therapy & $3.4 \pm 1.5$ & $4.4 \pm 1.8$ & - & - \\
\hline Final assessment & $2.4 \pm 1.1$ & $5.1 \pm 1.3$ & - & - \\
\hline Group (G) & - & - & 14.4 & $0.045^{*}$ \\
\hline Time (T) & - & - & 41.1 & $<0.001^{*}$ \\
\hline $\mathrm{G} \times \mathrm{T}$ & - & - & 8.2 & 0.706 \\
\hline Mauchly's sphericity test & \multicolumn{2}{|c|}{$\mathrm{W}=0.175$} & - & 0.071 \\
\hline \multicolumn{5}{|l|}{ VAS-A } \\
\hline Before first therapy & $7.0 \pm 1.5$ & $7.2 \pm 1.4$ & - & - \\
\hline After first therapy & $5.4 \pm 1.3$ & $6.4 \pm 1.1$ & - & - \\
\hline After third therapy & $4.1 \pm 1.0$ & $5.4 \pm 0.9$ & - & - \\
\hline Final assessment & $2.8 \pm 0.8$ & $5.8 \pm 2.0$ & - & - \\
\hline Group (G) & - & - & 15.0 & $0.010^{*}$ \\
\hline Time (T) & - & - & 63.3 & $<0.001^{*}$ \\
\hline $\mathrm{G} \times \mathrm{T}$ & - & - & 8.3 & 0.162 \\
\hline Mauchly's sphericity test & \multicolumn{2}{|c|}{$\mathrm{W}=0.320$} & - & 0.097 \\
\hline
\end{tabular}

Values are presented as mean \pm standard deviation.

ESWT, extracorporeal shock wave therapy; VAS, visual analogue scale; R, resting status; A, active status.

${ }^{*} \mathrm{p}<0.05$. 


\section{Change of VAS of resting status}

Sphericity on VAS-R revealed that the sphericity assumption was satisfied $(\mathrm{p}=0.071)$, with a significant difference in VAS- $R$ between the groups $(\mathrm{p}=0.045)$ and with time $(\mathrm{p}<0.001)$, although there was no reciprocal action effect $(\mathrm{p}=0.706)$. Contrast testing with time revealed a significant difference in VAS-R prior to commencement of treatment, and following the first and third treatments. A significant reduction in VAS-R was noted following the treatment. Although there was a significant difference in VAS- $\mathrm{R}$ between the groups, there was no reciprocal action effect. This indicated that treatment using ESWT is more effective than the treatment using TENS and desensitization technique, since the VAS-R of the ESWT group was found lower than that of the control group (Table 4).

\section{Change of VAS of active status}

Sphericity on VAS-A revealed that the sphericity assumption was satisfied $(\mathrm{p}=0.097)$, with a significant difference in VAS-A between the groups $(\mathrm{p}=0.010)$ and with time $(\mathrm{p}<0.001)$, although there was no reciprocal action effect $(p=0.162)$. Contrast testing with time revealed a significant difference in VAS-A prior to commencement of treatment, and following the first and third treatments. A significant reduction in VAS-A was noted following the treatment. Although there was a significant difference in VAS-A between the groups, there was no reciprocal action effect indicating that treatment using ESWT is more effective than the treatment using TENS and desensitization technique, since the VAS-A of the ESWT group was lower than that of the control group (Table 4).

\section{Change of PRS of resting status}

The sphericity assumption was satisfied as a result of repeated measure ANOVA on PRS- $\mathrm{R}(\mathrm{p}=0.456)$, with significant differences in PRS-R accordance between the groups $(\mathrm{p}=0.004)$, with time $(\mathrm{p}<0.001)$, and with reciprocal action $(\mathrm{p}=0.032)$. Contrast testing with time revealed a reduction following the first and third treatments in comparison to before treatment, illustrating that PRS-R was significantly reduced as the treatment was carried out. A significant difference was also evident in PRS-R between the groups. There was a significant difference in the contrast test results on reciprocal action prior to commence-

Table 5. Change of PRS of resting and active status

\begin{tabular}{|c|c|c|c|c|}
\hline & ESWT & Control & $\mathbf{F}$ & p-value \\
\hline \multicolumn{5}{|l|}{$\begin{array}{l}\text { PSR-R } \\
\text { PS }\end{array}$} \\
\hline Before first therapy & $40.2 \pm 15.5$ & $39.4 \pm 15.6$ & - & - \\
\hline After first therapy & $34.8 \pm 15.6$ & $36.7 \pm 15.3$ & - & - \\
\hline After third therapy & $28.0 \pm 13.5$ & $33.8 \pm 14.2$ & - & - \\
\hline Final assessment & $14.4 \pm 6.8$ & $38.3 \pm 16.0$ & - & - \\
\hline Group (G) & - & - & 39.5 & $0.004^{*}$ \\
\hline Time $(\mathrm{T})$ & - & - & 38.4 & $<0.001^{*}$ \\
\hline $\mathrm{G} \times \mathrm{T}$ & - & - & 15.4 & $0.032^{*}$ \\
\hline Mauchly's sphericity test & \multicolumn{2}{|c|}{$\mathrm{W}=0.128$} & - & 0.456 \\
\hline \multicolumn{5}{|l|}{ PSR-A } \\
\hline Before first therapy & $46.3 \pm 12.4$ & $44.2 \pm 15.4$ & - & - \\
\hline After first therapy & $37.6 \pm 9.9$ & $38.9 \pm 13.9$ & - & - \\
\hline After third therapy & $25.3 \pm 10.3$ & $33.3 \pm 12.0$ & - & - \\
\hline Final assessment & $15.8 \pm 6.0$ & $35.1 \pm 16.0$ & - & - \\
\hline Group (G) & - & - & 18.9 & $<0.001^{*}$ \\
\hline Time (T) & - & - & 59.6 & $0.010^{*}$ \\
\hline $\mathrm{G} \times \mathrm{T}$ & - & - & 9.9 & $0.018^{*}$ \\
\hline Mauchly's sphericity test & \multicolumn{2}{|c|}{$\mathrm{W}=0.236$} & - & 0.612 \\
\hline
\end{tabular}

Values are presented as mean \pm standard deviation.

ESWT, extracorporeal shock wave therapy; PRS, pain rating scale; $R$, resting status; A, active status.

${ }^{*} \mathrm{p}<0.05$. 
ment of treatment and following the third treatment indicating that although the PRS-R prior to commencement was higher in the ESWT group, it was lower in the ESWT group following the third treatment and that ESWT was more effective after the third treatment (Table 5).

\section{Change of PRS of active status}

The sphericity assumption was satisfied as a result of repeated measure ANOVA on PRS-A ( $\mathrm{p}=0.612)$, with significant differences in PRS-A accordance between the groups $(\mathrm{p}<0.001)$, with time $(\mathrm{p}=0.010)$, and with reciprocal action $(p=0.018)$. Contrast testing with time revealed a reduction following the first and third treatments in comparison to before treatment indicating that PRS-A was significantly reduced as the treatment was carried out. A significant difference in PRS-A between the groups was also evident. There was a significant difference in the results of the contrast test on reciprocal action prior to commencement and following the third treatment indicating that, although the PRS-A prior to commencement was higher in the ESWT group, it was lower in the ESWT group following the third treatment and that ESWT was more effective after the third treatment (Table 5).

\section{Change of pain threshold}

The sphericity assumption was unsatisfactory based on the result of sphericity on PRS ( $\mathrm{p}<0.001)$. Repeated measure ANOVA carried out by Wilks' lambda demonstrated no significant difference between the groups $(\mathrm{p}=0.586)$, no reciprocal action effect $(\mathrm{p}=0.644)$, but a significant effect with time $(p=0.005)$. Contrast testing with time revealed significant differences prior to commencement of treatment, and following the first and third treatments indicating that pain threshold was increased significantly after the first treatment (Table 6).

Table 6. Change of pain threshold

\begin{tabular}{|c|c|c|c|c|}
\hline & ESWT & Control & $\mathbf{F}$ & p-value \\
\hline Before first therapy & $7.3 \pm 2.8$ & $7.0 \pm 2.5$ & - & - \\
\hline After first therapy & $8.7 \pm 3.4$ & $8.0 \pm 2.9$ & - & - \\
\hline After third therapy & $11.1 \pm 3.4$ & $10.2 \pm 3.0$ & - & - \\
\hline Final assessment & $16.1 \pm 3.7$ & $16.4 \pm 3.1$ & - & - \\
\hline Group (G) & - & - & 59.6 & 0.586 \\
\hline Time $(\mathrm{T})$ & - & - & 62.0 & $<0.005^{*}$ \\
\hline $\mathrm{G} \times \mathrm{T}$ & - & - & 25.2 & 0.644 \\
\hline Mauchly's sphericity test & \multicolumn{2}{|c|}{$\mathrm{W}=0.130$} & - & $<0.001$ \\
\hline
\end{tabular}

Values are presented as mean \pm standard deviation.

ESWT, extracorporeal shock wave therapy.

${ }^{*} \mathrm{p}<0.05$.

Table 7. Change of neuroma size

\begin{tabular}{|c|c|c|c|c|}
\hline & ESWT & Control & $\mathbf{F}$ & p-value \\
\hline Before first therapy & $1.9 \pm 0.4$ & $1.9 \pm 0.4$ & - & - \\
\hline After first therapy & $1.9 \pm 0.5$ & $1.9 \pm 0.4$ & - & - \\
\hline After third therapy & $1.9 \pm 0.4$ & $1.8 \pm 0.4$ & - & - \\
\hline Final assessment & $1.9 \pm 0.4$ & $1.8 \pm 0.4$ & - & - \\
\hline Group (G) & - & - & 0.6 & 0.556 \\
\hline Time (T) & - & - & 0.8 & 0.460 \\
\hline $\mathrm{G} \times \mathrm{T}$ & - & - & 0.3 & 0.799 \\
\hline Mauchly's sphericity test & \multicolumn{2}{|c|}{$\mathrm{W}=0.002$} & - & $<0.001$ \\
\hline
\end{tabular}

Values are presented as mean \pm standard deviation.

ESWT, extracorporeal shock wave therapy. 


\section{Change of neuroma size}

The sphericity assumption was unsatisfactory based on the result of sphericity on PRS $(\mathrm{p}<0.001)$. Repeated measure ANOVA carried out by Wilks' lambda revealed no significant difference between the groups $(\mathrm{p}=0.556)$, with time ( $\mathrm{p}=0.460)$, and according to reciprocal action ( $\mathrm{p}=0.799)$ (Table 7).

\section{DISCUSSION}

The development of amputation stump neuromas is a common and frequent cause of stump pain. Neuromas may be thought of as a normal part of the healing process [2]. There are two types of stump neuromas: terminal neuromas and spindle neuromas. When a nerve is damaged, the peripheral Schwann cells in the distal portion of the nerve proliferate so that the proximal axons can grow into this new coat of Schwann cells and attempt to reunite. In an amputation, there are no distal Schwann cells left to form this coat. Thus, the proximal axons grow and form an extremely sensitive bulbous overgrowth referred to as a terminal neuroma [9]. Spindle neuromas are found away from the transected nerve ending in a peripheral nerve that has been exposed to microtrauma from stretching or compression by local scar tissue [2]. Neuropathic pain is influenced by the presence of me- chanical or chemical irritation, development of local scar tissue, traction neuritis, and dysesthetic sensory symptoms [10].

Various tools may be used to detect neuroma, but the most frequently used ones are magnetic resonance imaging and US. Using the latter, normal nerves are apparent as cord-like structures composed of echogenic linear striations on longitudinally oriented images and round echogenic structures on transverse images [11]. The classic appearance of a neuroma on US is an oval, hypoechoic mass in direct contiguity with the nerve. It can be either well defined or have irregular margins [1]. In addition to localization, real-time imaging allows for correlation with the clinical examination, often verifying the visualized lesion as the source of the pain or palpable mass [1]. US was involved in detecting neuroma with all patients in this study due to these advantages, and assessment of neuroma size was used to identify the relationship between size change and pain in determining the curative effect of ESWT. However, no statistical relevance was apparent between US-evident neuroma size change performed 3 months after treatment and treatment success rate (Fig. 3). These observations imply that a pain reduction mechanism manifest after low-energy ESWT includes an analgesic effect, regardless of changed neuroma size.
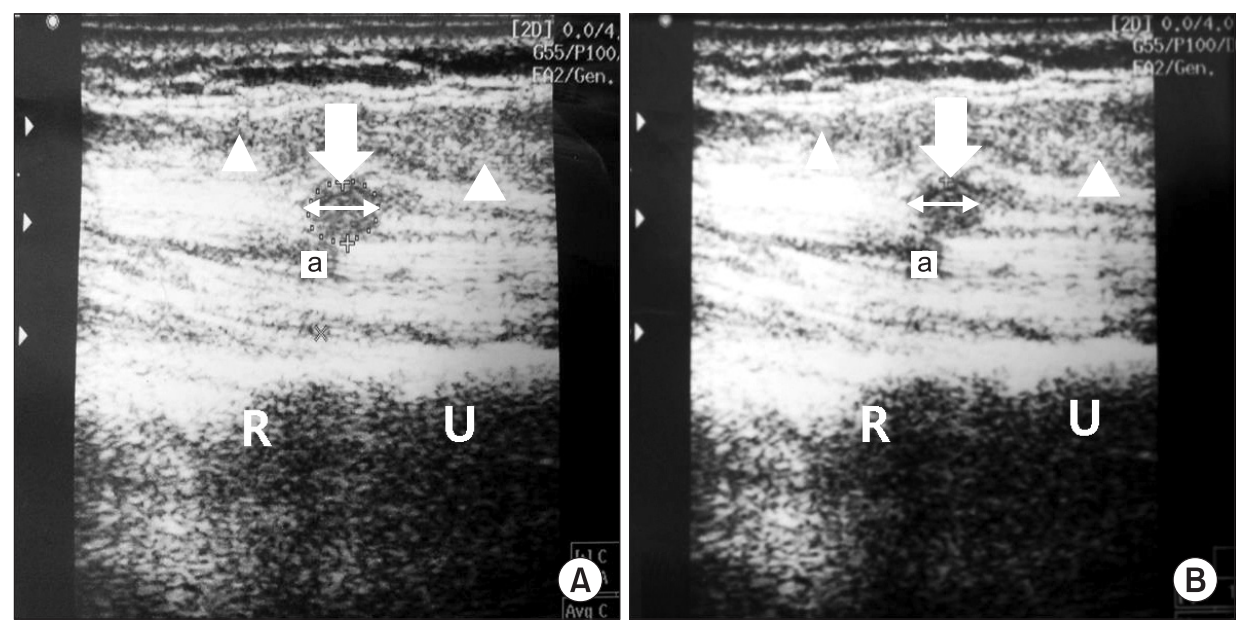

Fig. 3. A 43-year-old male (patient no. 7) with a transradial stump neuroma (arrow). Scanning over the proximal forearm demonstrates end stump of radius (R) and ulnar (U) bone. Transverse ultrasound image of proximal forearm showed hypoechoic mass (arrow) below the humeral head of pronator teres muscle (arrowhead) and close to the ulnar artery (a). No change of neuroma size was apparent before and following extracorporeal shock wave therapy (ESWT) treatment. (A) The neuroma of initial assessment before ESWT treatment. Digital calipers indicate the size of the neuroma is $10.6 \mathrm{~mm}$. (B) The neuroma at the final assessment after ESWT treatment. Digital calipers indicate the size of the neuroma is $10.8 \mathrm{~mm}$. 
Treatment of neuroma is difficult and has prompted extensive research into a wide variety of treatment options. Many techniques have been tried including percussion, implantation into muscle or bone, nerve-to-nerve anastomosis, local and free-flaps or venous nerve conduits, capping with inert materials, auterization, phenolization, steroid cryosurgery, and alcohol injections [12]. However, no technique had a definitive outcome and all were accompanied by side effects.

ESWT has been shown to be clinically effective in treating numerous musculoskeletal problems, including lateral epicondylitis, calcific tendonitis of the shoulder, and plantar fasciitis. In this study, we confirmed the diagnosis of stump neuroma through US examination on patients suspected of stump neuroma after clinical diagnosis among those complaining of chronic stump pain. Also, we were able to determine the most acute pain area and achieve effective ESWT application, because treatment was applied on the ESWT-applied area without topical anesthesia. Moreover, any disturbing variable affecting treatment results was excluded by not prescribing oral nonsteroidal anti-inflammatory drugs during the treatment and assessment periods.

This study employed $0.10 \mathrm{~mJ} / \mathrm{mm}^{2}$ low-energy shock waves. ESWT can be categorized into low-energy ( 0.08 to $0.27 \mathrm{~mJ} / \mathrm{mm}^{2}$ ), medium-energy ( 0.28 to $0.59 \mathrm{~mJ} / \mathrm{mm}^{2}$ ), and high-energy $\left(\geq 0.6 \mathrm{~mJ} / \mathrm{mm}^{2}\right)$ by EFD level. By another standard, energy density values $\leq 0.12 \mathrm{~mJ} / \mathrm{mm}^{2}$ for lowenergy and high-energy $\geq 0.12 \mathrm{~mJ} / \mathrm{mm}^{2}$ are suggested, but there is no clearly unified standard [13]. No guideline clearly defines the number of and interval for treating stump neuroma by ESWT. In one study [14], low-energy shock wave treatment for chronic epicondylitis of elbow joint was carried out, with approximately 2,000 shock waves with low EFD range of 0.06 to $0.12 \mathrm{~mJ} / \mathrm{mm}^{2}$ delivered in accordance with the pain tolerance of the patient during each treatment three times with a 1-week interval between treatments. Another study [15] on the effectiveness of ESWT on plantar fasciitis patients utilized 1,200 shock waves with low EFD of $0.24 \mathrm{~mJ} / \mathrm{mm}^{2}$ were also delivered three times at 1-week intervals. The subjects received a total of 1,500 shock waves for each treatment at the rate of 240 waves, each with low EFD of $0.10 \mathrm{~mJ} / \mathrm{mm}^{2}$ / $\mathrm{min}$ for three times with a 1-week treatment interval (total of 4,500 shock waves). We chose a low-energy density of $0.10 \mathrm{~mJ} / \mathrm{mm}^{2}$ considering the hypersensitive character- istics of neuroma and patient compliance, as well as the fact that direct appliance of ESWT to stump neuroma has a high probability of nerve damage.

In another study [16], 25 patients (25 feet) were recruited and consented to take part in a placebo-controlled and blinded study to evaluate the efficacy and safety of ESWT to ablate clinically confirmed Morton's neuroma. The active group was treated using a OssaTron device (HealthTronics, Austin, TX, USA) using 2,000 pulses delivered at $21 \mathrm{kV}$ inferior to the neuroma. The sham foot received no treatment. Fourteen subjects were randomized to the active group and 11 to the sham group. Posttreatment evaluations were carried out at 1, 6, and 12 weeks by a blinded investigator. End point evaluation parameters were reduction in VAS and Roles-Maudsley quality of life assessment. Sixty-four percent of the active group $(n=9)$ reported improvement exceeding $50 \%$, as compared with $27 \%$ of the sham group $(n=3)$. At 12 weeks post-treatment, $57 \%$ of the active group $(n=8)$ attained a VAS $<3$, while $27 \%$ of the sham group ( $n=3$ ) achieved a similar reduction. Seven percent of the active group $(n=1)$ had no improvement, while $64 \%$ of the sham group $(n=7)$ had no improvement. There were no complications in either group. However, there was no mention of how the treatment effect of ESWT was applied to neuroma. Also, video examinations including US and magnetic resonance imaging to verify the effects of ESWT on neuroma were not used to determine the effects of diagnosis tools or treatments, whereas the determination of neuroma treatment area was also focused on patient symptoms without US guidance.

To examine the application of ESWT to stump neuroma in this study, stump pain results were first assessed from nerve traction by scar tissue surrounding and fixing the neuroma [6], from compression of the sensitive nerve endings by adjacent soft tissues, or from reduced blood supply to the neuroma with hypoxia of the nerve endings [17]. Peripheral up-regulation of various ion channels and receptors (sodium channels, transient receptor potential channel 1 , calcitonin gene-related peptide, $\alpha_{1 C}$ receptors, and nerve growth factor) may also contribute to the neuropathic pain [18]. The delivery of shock waves to scar tissue when ESWT is applied to stump neuroma is thought to cause microscopic injury that breaks down collagen fibers, enabling scar remodeling [19]. The remodeling of scars surrounding a neuroma may release 
tissues in compression or traction around the neuroma to induce pain relief. Additionally, patients complained about pain more with active stump neuroma status than resting status, supporting the hypothesis that scar tissues fixing neuroma in active status cause neuroma traction during patient movement, which causes even more acute pain. Scar remodeling may also contribute even more for stump neuroma pain reduction in active status on that basis.

In addition, a study [20] comparing a group of rat skins undergoing ESWT prior to flap operation and another group without ESWT prior to flap operation showed that early stage benefits included effects, such as vasodilatation, and an increased expression of angio-active factors, such as nitric oxide and vascular endothelial growth factor, with the late stage characterized by significant increases of visible collateral vessels and microvessel density. The authors concluded that an increased blood supply at early stage provides the flap with a minimum of nutrition and oxygen to keep the ischemic flap tissue alive until the formation of new vessels. Furthermore, the authors found a significant increase in vessel density in the late stage. We think that neuroma ESWT may reduce hypoxia by an angiogenesis effect caused by early stage and late stage dilatation of vessels around the neuroma to generate pain relief in both early and late stages because nerve ending hypoxia was caused by reduction of blood supply to stump neuroma.

Additionally, a study [21] in which ESWT was used for myofascial pain syndrome indicated the generation of a mechanism controlling associated pain by improving local ischemia and controlling metabolic increase and insufficient energy due to local ischemia, reduced secretion of various pain-causing materials, prevention of central sensitization, and control of excessive excitement of neurons due to the reduction of substance $P$ synthesis. Such a mechanism may play a collateral role to relieve pain when ESWT is applied to stump neuroma as well.

We have demonstrated no complications with this technique. It prevents many complications normally associated with surgical excision, as well as the time required to recover from surgery. Hence, we continue to offer ESWT as an alternative to surgical excision for stump neuroma. Although these results are very encouraging, potentially reducing the need for surgical excision, we would recommend long-term studies with larger numbers of patients.
An animal study is essential for investigation of pathophysiology of pain reducing effects in ESWT.

In conclusion, ESWT has been proposed in several studies as an alternative treatment method that can be applied to musculoskeletal disorder in various ways as a noninvasive treatment method with few side effects. The present results support the value of ESWT on stump neuroma compared to other treatment methods employed widely now. Moreover, we verified the application of ESWT has advantages besides treatment effects as it can reduce side effects generated through injection therapies and surgical treatments. We believe it is important to establish standard treatment instructions by performing more treatment/control comparisons.

\section{CONFLICT OF INTEREST}

No potential conflict of interest relevant to this article was reported.

\section{ACKNOWLEDGMENTS}

This study was supported by a grant of the Korea Health Technology R\&D Project, Ministry of Health \& Welfare, Republic of Korea (A120942) and by Basic Science Research Program through the National Research Foundation of Korea (NRF) funded by the Ministry of Education (NRF-2014R1A1A4A01007956).

\section{REFERENCES}

1. Provost N, Bonaldi VM, Sarazin L, Cho KH, Chhem RK. Amputation stump neuroma: ultrasound features. J Clin Ultrasound 1997;25:85-9.

2. Henrot P, Stines J, Walter F, Martinet N, Paysant J, Blum A. Imaging of the painful lower limb stump. Radiographics 2000;20 Spec No:S219-35.

3. Ernberg LA, Adler RS, Lane J. Ultrasound in the detection and treatment of a painful stump neuroma. Skeletal Radiol 2003;32:306-9.

4. Vernadakis AJ, Koch H, Mackinnon SE. Management of neuromas. Clin Plast Surg 2003;30:247-68.

5. Whipple RR, Unsell RS. Treatment of painful neuromas. Orthop Clin North Am 1988;19:175-85.

6. Thomas AJ, Bull MJ, Howard AC, Saleh M. Peri operative ultrasound guided needle localisation of amputa- 
tion stump neuroma. Injury 1999;30:689-91.

7. Liang HW, Wang TG, Chen WS, Hou SM. Thinner plantar fascia predicts decreased pain after extracorporeal shock wave therapy. Clin Orthop Relat Res 2007;460:219-25.

8. Flor H. Phantom-limb pain: characteristics, causes, and treatment. Lancet Neurol 2002;1:182-9.

9. Fisher GT, Boswick JA Jr. Neuroma formation following digital amputations. J Trauma 1983;23:136-42.

10. Dockery GL. The treatment of intermetatarsal neuromas with $4 \%$ alcohol sclerosing injections. J Foot Ankle Surg 1999;38:403-8.

11. Sobiesk GA, Wertheimer SJ, Schulz R, Dalfovo M. Sonographic evaluation of interdigital neuromas. J Foot Ankle Surg 1997;36:364-6.

12. Wu J, Chiu DT. Painful neuromas: a review of treatment modalities. Ann Plast Surg 1999;43:661-7.

13. Speed CA. Extracorporeal shock-wave therapy in the management of chronic soft-tissue conditions. J Bone Joint Surg Br 2004;86:165-71.

14. Jung KH, Hwang JH, Chang HJ, Yoon YC, Park MJ, Yoo JC, et al. Low-energy extracorporeal shock wave therapy on chronic epicondylitis of the elbow: clinical and sonographic study. J Korean Acad Rehabil Med 2009; 33:77-83.

15. Kim SB, Lee KW, Lee JH, Kim YD, Yoon K, Joe YL. The effect of extracorporeal shock wave therapy in plantar fasciitis. J Korean Acad Rehabil Med 2009;33:333-8.

16. Fridman R, Cain JD, Weil L Jr. Extracorporeal shockwave therapy for interdigital neuroma: a randomized, placebo-controlled, double-blind trial. J Am Podiatr Med Assoc 2009;99:191-3.

17. Mathews GJ, Osterholm JL. Painful traumatic neuromas. Surg Clin North Am 1972;52:1313-24.

18. Hagenacker T, Ledwig D, Busselberg D. Feedback mechanisms in the regulation of intracellular calcium $([\mathrm{Ca} 2+] \mathrm{i})$ in the peripheral nociceptive system: role of TRPV- 1 and pain related receptors. Cell Calcium 2008;43:215-27.

19. Fioramonti P, Cigna E, Onesti MG, Fino P, Fallico N, Scuderi N. Extracorporeal shock wave therapy for the management of burn scars. Dermatol Surg 2012;38:778-82.

20. Keil H, Mueller W, Herold-Mende C, Gebhard MM, Germann G, Engel H, et al. Preoperative shock wave treatment enhances ischemic tissue survival, blood flow and angiogenesis in a rat skin flap model. Int J Surg 2011;9:292-6.

21. Jeon JH, Jung YJ, Lee JY, Choi JS, Mun JH, Park WY, et al. The effect of extracorporeal shock wave therapy on myofascial pain syndrome. Ann Rehabil Med 2012;36:665-74. 